\title{
Novas referências de hepáticas (Marchantiophyta) da Floresta Nacional de Caxiuanã para o Estado do Pará, Brasil
}

\author{
Lisi Dámaris Pereira Alvarenga ${ }^{1,4}$, Regina Célia Lobato Lisboa² e Ana Cláudia Caldeira Tavares ${ }^{3}$
}

Recebido em 19/06/2006. Aceito em 21/12/2006

\begin{abstract}
RESUMO - (Novas referências de hepáticas (Marchantiophyta) da Floresta Nacional de Caxiuanã para o Estado do Pará, Brasil). São apresentadas no presente trabalho oito hepáticas referidas pela primeira vez para o Estado do Pará, sendo cinco delas novas referências também para a região Norte do país. Todas são provenientes da Estação Científica Ferreira Penna (ECFPn) (142’30”'S e 51³1’45”'W), localizada na Floresta Nacional de Caxiuanã (Flona Caxiuanã, Amazônia Oriental). São elas Ceratolejeunea desciscens (Sande-Lac.) Schiffn., Colura greig-smittii Jovet-Ast, Lejeunea raddiana Lindenb. Gottsche, Rectolejeunea emarginuliflora (Gottsche) A. Evans, Arachniopsis monodactyla (Spruce) R.M. Schust., Plagiochila aerea Tayl., Plagiochila gymnocalycina (Lehm. \& Lindenb.) Lindenb. e Plagiochila martiana (Nees) Lindenb. São fornecidos comentários taxonômicos relevantes e ilustrações das espécies.
\end{abstract}

Palavras-chave: briófitas, brioflora, Estação Científica Ferreira Penna, Amazônia Oriental

\begin{abstract}
New liverwort records for the Caxiuanã National Forest Pará State, Brazil). Eight new records of liverwort species for Pará state are reported; five of these are also new records for Northern Brazil. These species come from the Ferreira Penna Scientific Station (1 $1^{\circ} 42^{\prime} 30^{\prime \prime}$; ; 51 $1^{\circ} 31^{\prime} 45^{\prime \prime} \mathrm{W}$ ) located in Caxiuanã National Forest (Eastern Amazonia). They are Ceratolejeunea desciscens (SandeLac.) Schiffn., Colura greig-smittii Jovet-Ast, Lejeunea raddiana Lindenb. Gottsche, Rectolejeunea emarginuliflora (Gottsche) A. Evans, Arachniopsis monodactyla (Spruce) R.M. Schust., Plagiochila aerea Tayl., Plagiochila gymnocalycina (Lehm. \& Lindenb.) Lindenb. and Plagiochila martiana (Nees) Lindenb. Relevant taxonomic comments are included as well as species illustrations.
\end{abstract}

Key words: bryophytes, bryoflora, Ferreira Penna Scientific Station, Eastern Amazonia

\section{Introdução}

O conhecimento sobre a brioflora brasileira teve início no começo de século XIX com o trabalho de naturalistas que realizavam expedições de coleta principalmente nas regiões Sul, Sudeste e Norte do país (Pôrto 1996; Gradstein \& Costa 2003).

O primeiro relato de briófitas para o Brasil foi feito por Raddi, em 1820, que publicou uma nova espécie de musgo, porém os estudos de maior importância para a flora briológica brasileira só apareceram a partir de 1830 com os trabalhos de Nees von Esenbeck, Martius, Montagne, Hornschuch, Müller, Hampe, Spruce, Mitten, entre outros (Vital 1969).

Ao longo de pouco mais de 200 anos, o conhecimento sobre a flora briofítica no Brasil tem aumentado bastante, no entanto quando se considera as áreas ainda não estudadas observa-se a necessidade de intensificar os trabalhos de coleta, principalmente quando se refere à Amazônia.

Segundo Lisboa (1991) os estudos de briófitas na Amazônia podem ser divididos em dois períodos: $1^{\circ}$ Período dos Grandes Naturalistas, iniciado com Martius e outros grandes naturalistas europeus; $2^{\circ}$ - Período dos Briologistas, iniciado por volta de $1970 \mathrm{com}$ as primeiras coletas de G. T. Prance e a vinda do briólogo Dana Griffin III para a Amazônia, além de outros pesquisadores do Projeto Flora Amazônica. Este período estende-se até os dias atuais.

Diversos levantamentos vêm sendo realizados na região amazônica, sendo a maioria deles incluídos no Projeto Briófitas do Estado do Pará, o qual dispõe de informações sobre vários municípios deste Estado que, entre outros, podem ser encontradas em: Lisboa (1993; 1994), Lisboa \& Ilkiu-Borges (1997a; b), Lisboa \& Nazaré (1997; 2002), Lisboa et al. (1999), Lisboa \&

\footnotetext{
1 Universidade Federal de Pernambuco, CCB, Departamento de Botânica, Rua Prof. Moraes Rego s.n., Cidade Universitária, 50670-420 Recife, PE, Brasil (lisidamaris@yahoo.com.br)

2 Museu Paraense Emílio Goeldi, C. Postal 399, 66040-170 Belém, PA, Brasil (regina@museu-goeldi.br)

3 Instituto de Pesquisas Jardim Botânico do Rio de Janeiro, Rua Pacheco Leão, 2040, Horto, 20460-430 Rio de Janeiro, RJ, Brasil (anatavares@jbrj.gov.br)

4 Autor para correspondência: lisidamaris@yahoo.com.br
} 
Ilkiu-Borges (2001), Ilkiu-Borges \& Lisboa (2002a; b; c; d; 2004), Santos \& Lisboa (2003), Osakada \& Lisboa (2004), Lisboa \& Santos (2005a; b), Souza \& Lisboa (2005). Nesses trabalhos observa-se sempre registros de novas ocorrências para o Estado do Pará, e em alguns deles até para a Amazônia e Brasil, fato já previsto em Lisboa (1994) onde ressaltou que à medida que fossem realizados mais trabalhos de coletas, novos registros seriam encontrados.

O presente trabalho objetivou ampliar o conhecimento sobre a brioflora através do registro de oito espécies de hepáticas para o Estado do Pará.

\section{Material e métodos}

O material é proveniente da Estação Científica Ferreira Penna (ECFPn) que se localiza no interior da Floresta Nacional de Caxiuanã (FLONA de Caxiuanã, 142’30’S $51^{\circ} 31^{\prime} 45^{\prime}$ 'W), município de Melgaço, Pará. A cobertura vegetal em Caxiuanã apresenta ecossistemas típicos da floresta amazônica, possuindo áreas de mata de terra firme, mata de inundação (várzea e igapó) trechos de vegetação secundária (capoeira) de diversas idades e trechos de vegetação savanóide (campinas amazônicas) (Lisboa 2002).

As coletas de briófitas foram realizadas de acordo com Lisboa (1993), retirando-se, com auxílio de facão, as populações dos substratos aos quais estão na maioria das vezes firmemente aderidas, quando possível retirando juntamente parte destes para a melhor preservação da estrutura original das plantas. Os espécimes foram acondicionados em sacos de papel de $1 \mathrm{~kg}$ ou $2 \mathrm{~kg}$. Em seguida à coleta, o material foi seco dentro dos sacos ao sol ou em estufa de campo $\left(40-60{ }^{\circ} \mathrm{C}\right)$ quando necessário, por exemplo, em caso de material muito úmido, como briófitas em galhos submersos.

Em laboratório, o material botânico foi examinado ao estereomicroscópio para observação do hábito e confecção de lâminas visando à observação ao microscópio de luz. Para a identificação específica e o embasamento dos comentários taxonômicos fornecidos nos resultados foi utilizada, basicamente, a seguinte bibliografia: Evans (1906), Jovet-Ast (1953), Fulford (1968), Gradstein (1994), Heinrichs et al. (1998), Grolle \& Heinrichs (1999), Reiner-Drehwald \& Goda (2000), Gradstein et al. (2001), Gradstein \& Costa (2003), Dauphin (2003), Bastos (2004). A distribuição geográfica foi baseada em Gradstein \& Costa (2003) e nos catálogos de Yano $(1981 ; 1984 ; 1989 ; 1995)$. O sistema de classificação seguido foi o contido em
Crandall-Stotler \& Stotler (2000) para hepáticas e Buck $\&$ Goffinet (2000) para musgos. Todas as amostras encontram-se registradas no herbário "João Murça Pires" (MG) do Museu Paraense Emílio Goeldi.

\section{Resultados e discussão}

As oito hepáticas (Marchantiophyta) que são referidas pela primeira vez para o estado do Pará, são brevemente comentadas e ilustradas a seguir. As espécies que são novas ocorrências para a região Norte estão assinaladas com asterisco.

\section{MARCHANTIOPHYTA}

\section{LEJEUNEACEAE}

Ceratolejeunea desciscens (Sande-Lac.) Schiffn., Engler \& Prantl., Nat. Pflanzenfam. 1(3): 126. 1893. Tipo. Suriname. Sem localidade, Focke s.n. (Holótipo, L).

Fig. 1-3

Esta é caracterizada pelo anfigastro inteiro, lóbulos tipicamente inflados na porção inferior e planos em direção à margem livre e perianto com cornos bulbosos. C. desciscens é a espécie mais comum do subgênero e se diferencia por apresentar (1)2-6 ocelos em fileira, diferindo de $C$. grandiloba Jack \& Steph. e C. szyszylowiczii (Loitl.) Steph. que apresentam ocelos espalhados na lâmina, e pela margem do filídio inteira, diferindo de $C$. globulifera Herz. que possui margens denteadas. É encontrada, geralmente, como epífita em ambientes abertos como copas das árvores, e é registrada apenas para a Bacia Amazônia (floresta de planície) e para os Andes (até $1.400 \mathrm{~m}$ ). Na ECFPn ocorreu sobre tronco vivo e tronco morto apenas em mata de terra firme.

Descrição detalhada: Dauphin (2003).

Distribuição geográfica: Neotropical. No Brasil: AM (Dauphin 2003).

Material examinado: BRASIL. Pará: Município de Melgaço, Floresta Nacional de Caxiuanã, ECFPn, trilha para o Plot 1 do projeto TEAM, mata de terra firme, sobre tronco vivo, 18/X/2004, L.D.P. Alvarenga 202 (MG); ibidem, Plot 6 do projeto TEAM, mata de terra firme, sobre tronco morto, 5/XI/2004, L.D.P. Alvarenga 465 (MG).

Colura greig-smithii Jovet-Ast, Rev. Bryol. Lichénol. 22: 293. 1953. Tipo. Trinidad. Blanchisseuse Road. $P$. Greig-Smith (Holótipo, PC).

Fig. 4-7. 
É uma espécie tipicamente epífila, como é comum no gênero, e apresenta as células do saco inflado mamilosas por projeções cônicas e saco oblongo a ovalado não mais que duas vezes mais longo que largo. C. greig-smithii se assemelha a C. tortifolia (Nees $\&$ Mont.) Steph., sendo que esta última possui o saco com forma cilíndrica e até quatro vezes mais longo que largo. C. greig-smithii ocorre, geralmente, em florestas tropicais e é registrada apenas para a América do sul em elevações desde o nível do mar até $700 \mathrm{~m}$. $\mathrm{Na}$ ECFPn ocorreu sobre folha em mata de várzea e mata de terra firme.

Descrição detalhada: Jovet-Ast (1953).

Distribuição geográfica: América do Sul. No Brasil: AM, PE e SP (Gradstein \& Costa 2003; Germano \& Pôrto 2004).

Material examinado: BRASIL. Pará: Município de Melgaço, Floresta Nacional de Caxiuanã, ECFPn, igarapé Curuá, mata de várzea, sobre folha, 20/X/2004, L.D.P. Alvarenga 226 (MG); ibidem, trilha para o Plot 2 do projeto TEAM, mata de terra firme, sobre folha, 1/XI/2004, L.D.P. Alvarenga 392 (MG).

*Lejeunea raddiana Lindenb. Gottsche, Lindenb. \& Nees, Syn. Hepat.: 342. 1845. Tipo. Brasil. Raddi s.n. (holótipo, W [Lindenb. Hep. 6306]; isótipo, G 18049).

Fig. 8-10

Esta espécie caracteriza-se pelas margens dos filídios e dos periantos conspicuamente crenuladas, filídios geralmente apiculados e anfigastros pequenos. L. raddiana ocorre preferencialmente em madeira em decomposição e possui registro apenas para a Serra do Mar no Brasil (até $70 \mathrm{~m}$ ) e para a Bolívia (até $700 \mathrm{~m}$ ). Na ECFPn ocorreu como epífita e epíxila em mata de terra firme.

Descrição detalhada: Reiner-Drehwald \& Goda (2000).

Distribuição geográfica: Brasil e Bolívia. No Brasil: RJ, SP e RS (Reiner-Drehwald \& Goda 2000; Gradstein \& Costa 2003)

Material examinado: BRASIL. Pará: Município de Melgaço, Floresta Nacional de Caxiuanã, ECFPn, trilha para o Plot 5 do projeto TEAM, mata de terra firme, sobre raiz de tronco vivo, 9/XI/2004, L.D.P. Alvarenga 496 (MG); ibidem, sobre tronco morto, 9/XI/2004, L.D.P. Alvarenga 505 (MG); ibidem, Plot 3 do projeto TEAM, mata de terra firme, sobre tronco morto, 12/XI/2004, L. D. P. Alvarenga 588 (MG).
*Rectolejeunea emarginuliflora (Gottsche) A.Evans, Bull. Torrey Bot. Club 33: 14. 1906. Tipo. Cuba s/col, s/d, Wright 55.

Fig. 11-13

A presença de filídios caducos poucos modificados e produzidos em ramos ordinários e ocelos em menor número diferencia $R$. emarginuliflora de $R$. berteroana (Gottsche ex Steph.) A. Evans, a espécie mais comum do gênero, que produz os filídios caducos menores e em ramos flageliformes especializados. Ambas compartilham, contudo, o caráter dióico da inflorescência, o que as separa de $R$. monoica R.M. Schust. Outros caracteres típicos de $R$. emarginuliflora são as gemas talóides nas margens dos filídios caducos, ou protonemas conforme denomina Evans (1906), e ocelos basais pouco conspícuos, laminares e indistintos. A espécie geralmente ocorre como epífita em florestas ombrófilas e cerrado litorâneo. Na ECFPn ocorreu sobre tronco vivo em mata de terra firme e mata de várzea.

Descrição detalhada: Evans (1906).

Distribuição geográfica: Cuba, Porto Rico e Brasil. No Brasil: BA e SP (Gradstein \& Costa 2003; Bastos \& Yano 2003).

Material examinado: BRASIL. Pará: Município de Melgaço, Floresta Nacional de Caxiuanã, ECFPn, trilha para o Plot 1 do projeto TEAM, mata de terra firme, sobre tronco vivo, 18/X/2004, L.D.P. Alvarenga 202 (MG); ibidem, igarapé Curuá, mata de várzea, sobre tronco vivo, 20/X/2004, L.D.P. Alvarenga 221 (MG).

\section{LEPIDOZIACEAE}

Arachniopsis monodactyla (Spruce) R.M.Schust. Nova Hedwigia 10: 30. 1965. Tipo. Brasil. S. Gabriel. Spruce, Hep. Scpruc.; fl. Negro, Cataracas, (Isótipo, NY); S. Gabriel e Panduré, Spruce, Hep. Spruc. (BM, G-13418, NY).

Fig. 14

É uma espécie bastante pequena e delicada em comparação com outras espécies de hepáticas, composta de caulídios com quatro a cinco fileiras de células e filídios bastante rudimentares consistindo de 1(2)-4 células com uma papila na extremidade. A. monodactyla é registrada para as Guianas, Colômbia, Costa Rica e Brasil e ocorre geralmente sobre madeira em decomposição e solo em sítios muito úmidos de florestas tropicais. Na ECFPn ocorreu em tronco morto e tronco vivo em mata de terra firme. 


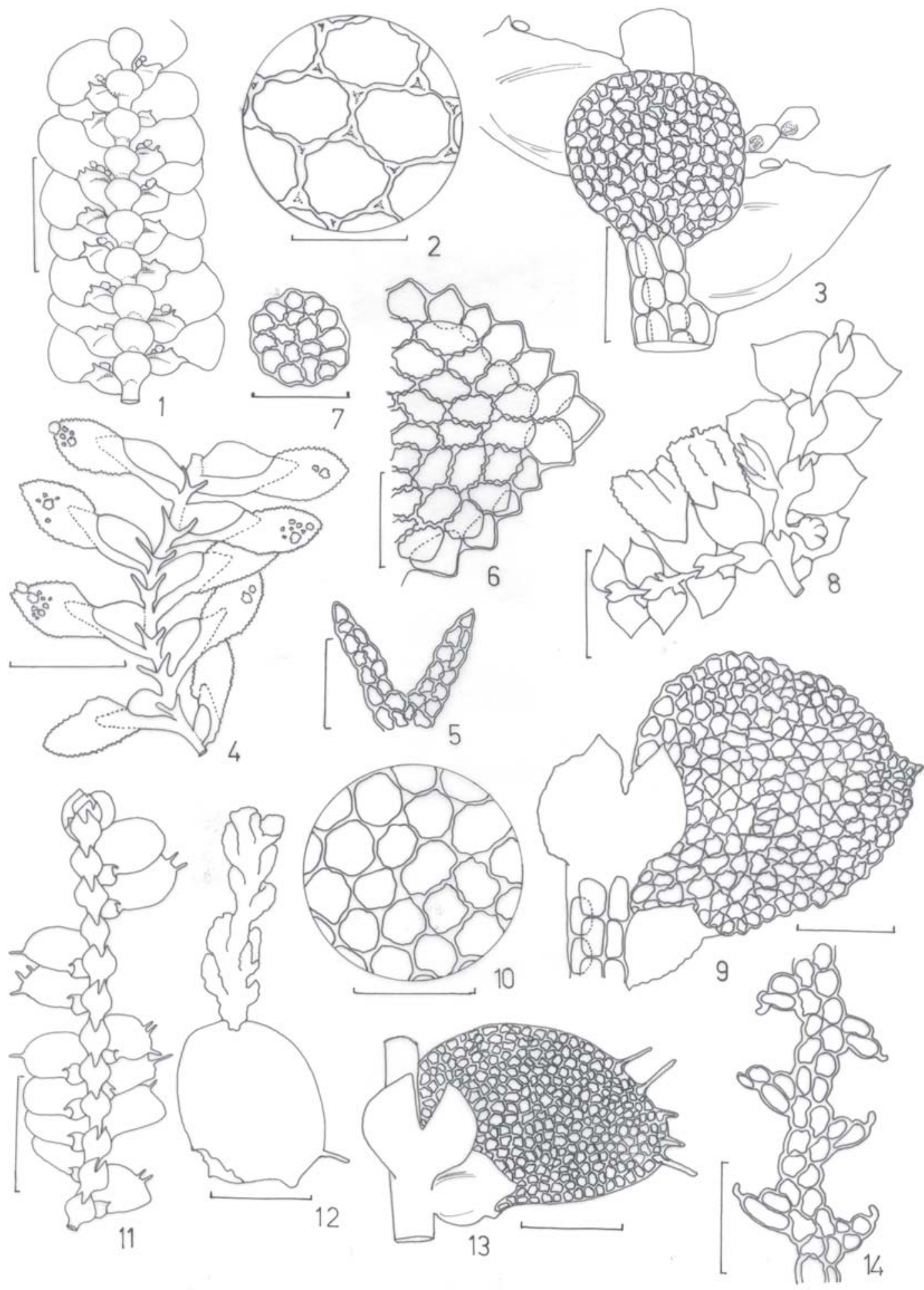

Figuras 1-3. Ceratolejeunea desciscens (Sande Lac.) Steph.1. Aspecto geral do gametófito, vista ventral. 2. Células medianas do filídio. 3. Anfigastro e lóbulo em detalhe. 4-7. Colura greig-smittii Jovet-Ast 4. Aspecto geral do gametófito, vista ventral 5. Anfigastro. 6. Ápice do filídio em detalhe. 7. Gema. 8-10. Lejeunea raddiana Lindenb. 8. Aspecto geral do gametófito, vista ventral. 9. Filídio e anfigastro em detalhe. 10. Células medianas do filídio. 11-13 Rectolejeunea emarginuliflora (Gottsche) A. Evans 11. Aspecto geral do gametófito, vista ventral. 12. Filídeo caduco. 13. Filídio e anfigastro em detalhe. 14. Arachniopsis monodactyla (Spruce) R.M. Schust. Aspecto geral do gametófito. Barras $=0,9 \mathrm{~mm}(1,8) ; 0,6 \mathrm{~mm}(4,11) ; 144 \mu \mathrm{m}(3) ; 120 \mu \mathrm{m}(5,9,12) ; 60 \mu \mathrm{m}(6,7) ; 30 \mu \mathrm{m}(2,10)$. 
Descrição detalhada: Fulford (1968).

Distribuição geográfica: Neotropical. No Brasil: AM e ES.

Material examinado: BRASIL. Pará: Município de Melgaço, Floresta Nacional de Caxiuanã, ECFPn, Plot 5 do projeto TEAM, mata de terra firme, sobre tronco morto, 9/XI/2004, L.D.P. Alvarenga 528 (MG); ibidem, trilha para o Plot 3 do projeto TEAM, mata de terra firme, sobre tronco morto, 12/XI/2004, L.D.P. Alvarenga 560 (MG); ibidem, plot 1 do projeto TEAM, mata de terra firme, sobre raiz de tronco vivo, 16/XI/2004, L.D.P. Alvarenga 616 (MG).

\section{PLAGIOCHILACEAE}

*Plagiochila aerea Tayl., London J. Bot. 5: 263. 1846. Tipo. Equador, Esmeralda 1827, Jamerson, s.n. "Greville Herb" (holótipo, E 00002817 [c. per., male]; Isótipo MANCH CC 7242, NY [c.per.], SW.

Fig. 15-17

Varia bastante em suas características, mas possui como peculiaridades mais distintivas os filídios linearoblongos com dentes ciliados a lanceolados, geralmente com dois dentes bastante conspícuos no ápice, e as células da lâmina fortemente alongadas e com paredes espessas. P. aerea era registrada até então como epífita em florestas nebulosas. Na ECFPn ocorreu sobre tronco vivo em mata de várzea.

Descrição detalhada: Grolle \& Heinrichs (1999).

Distribuição geográfica: Neotropical. No Brasil: DF (Gradstein \& Costa 2003).

Material examinado: BRASIL. Pará: Município de Melgaço, Floresta Nacional de Caxiuanã, ECFPn, igarapé Curuá, mata de várzea, sobre tronco vivo, 20/X/2004, L.D.P. Alvarenga 219 (MG).

*Plagiochila gymnocalycina (Lehm. \& Lindenb.) Lindenb. D’Orbigny, Voy Amer. Mér. 7, Bot. 2: 81. 1839.

Fig. $18-20$

Caracteriza-se pelos filídios alongados e voltados para o lado ventral do caulídio, periantos nus e inférteis, com a boca finamente e densamente ciliada e invólucro feminino muito reduzido, isto é, periantos não envoltos por brácteas. Heinrichs et al. (1998) consideram $P$. gymnocalycina sinônimo de $P$. simplex (Sw.) Lindenb. mas, conforme comentam Gradstein \& Costa (2003), esta última possui filídios menos alongados. $P$. gymnocalycina ocorre geralmente em tronco vivo e rochas em sítios úmidos de florestas montanas e formações arbustivas em elevações de 500 a 2.400 m.
Na ECFPn ocorreu sobre tronco vivo, eventualmente na serrapilheira, em mata de terra firme.

Descrição detalhada: Heinrichs et al. (1998).

Distribuição geográfica: Neotropical. No Brasil: PE, MG, RJ, SP e SC (Gradstein \& Costa 2003).

Material examinado: BRASIL. Pará: Município de Melgaço, Floresta Nacional de Caxiuanã, ECFPn, trilha para o Plot 1 do projeto TEAM, mata de terra firme, sobre tronco vivo, 18/X/2004, L.D.P. Alvarenga 210 (MG); ibidem, trilha para o Plot 4 do projeto TEAM ,mata de terra firme, sobre tronco vivo, 22/X/2004, L.D.P. Alvarenga $275(\mathrm{MG})$; ibidem, sobre tronco vivo, 26/X/2004, L.D.P. Alvarenga 296 (MG); ibidem, trilha para a torre de observação de $56 \mathrm{~m}$, mata de terra firme, sobre tronco vivo, 31/X/2004, L.D.P. Alvarenga 366 (MG); ibidem, trilha para o Plot 2 do projeto TEAM, mata de terra firme, sobre tronco vivo, 1/XI/2004, L.D.P. Alvarenga 376 (MG); sobre tronco vivo, $1 / \mathrm{XI} / 2004$, L.D.P. Alvarenga 380 (MG); ibidem, Plot 5 do projeto TEAM, mata de terra firme, sobre solo, 9/XI/2004, L.D.P. Alvarenga 537 (MG); ibidem, trilha para o Plot 1 do projeto TEAM, mata de terra firme, sobre raiz de tronco vivo, 16/XI/2004, L.D.P. Alvarenga 606 (MG).

* Plagiochila martiana (Nees) Lindenb. Sp. Hepat.: 75. 1840. Tipo. Brasil, col. Martius s.n.

Fig. 21-23

Caracteriza-se pelos filídios imbricados com margem ventral curto-decurrente e dorsal longodecurrente e as margens denteadas, assemelhando-se a $P$. patula sendo que esta última é bem mais robusta e possui a base da margem ventral conspicuamente longo decurrente. P. martiana ocorre geralmente sobre tronco vivo em florestas de planície e submontanas desde o nível do mar até $1.100 \mathrm{~m}$. Na ECFPn ocorreu sobre tronco vivo, algumas vezes semi-imerso, em mata de terra firme e mata de várzea.

Descrição detalhada: Gradstein \& Costa (2003).

Distribuição geográfica: Neotropical. No Brasil: PE, MG, RJ, SP, SC, PR e RS (Gradstein \& Costa 2003).

Material examinado: BRASIL. Pará: Município de Melgaço, Floresta Nacional de Caxiuanã, ECFPn, trilha para o Plot 5 do projeto TEAM, mata de terra firme, sobre tronco vivo, 9/XI/2004, L.D.P. Alvarenga 492 (MG); ibidem, entrada do Igarapé Santa Rosa, vegetação de várzea, sobre galho semi-imerso, 29/X/2004, L.D.P. Alvarenga 344 (MG); ibidem, sobre galho caído, 29/X/2004, L.D.P. Alvarenga 346 (MG); ibidem, sobre tronco vivo, 29/X/2004, L.D.P. 

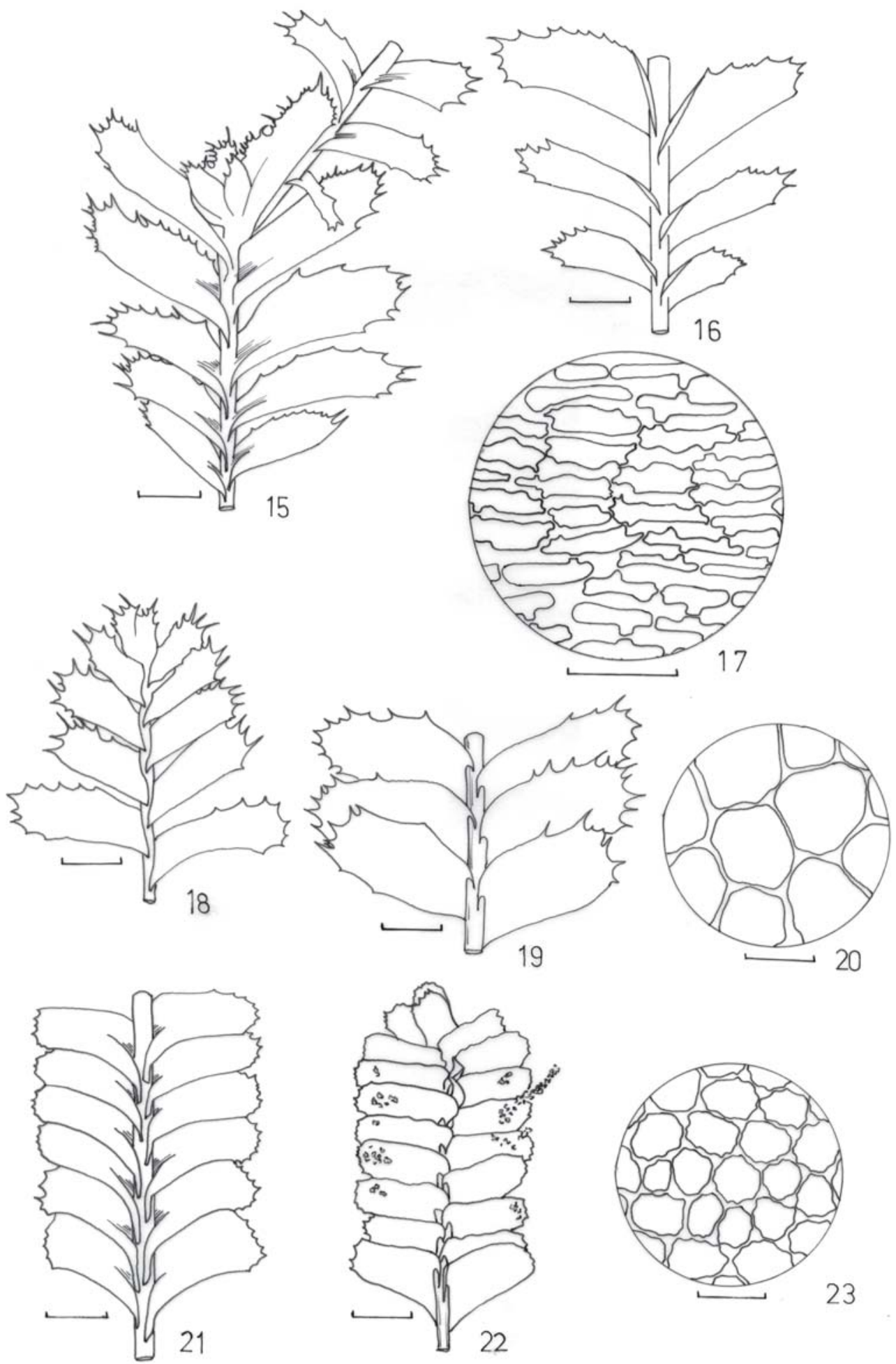

Figuras 15-17. Plagiochila aerea Tayl. L. 15. Gametófito, vista dorsal. 16. Gametófito, vista ventral. 17. Células medianas do filídio. 18-20. Plagiochila gymnocalycina (Lehm. \& Lindenb.) Lindenb. 18. Gametófito, vista dorsal. 19. Gametófito, vista ventral. 20. Células medianas do filídio. 21-23. Plagiochila martiana (Nees) Lindenb. 21. Gametófito, vista dorsal. 22. Gametófito, vista ventral. 23. Células medianas do filídio. Barras = 0,9 mm (14, 15, 18, 19, 21, 22); $90 \mu \mathrm{m}(16) ; 30 \mu \mathrm{m}(20,23)$. 
Alvarenga 347 (MG); ibidem, Praia do Lisboa, vegetação de várzea, sobre tronco vivo, 29/X/2004, L.D.P. Alvarenga 354 (MG); ibidem, sobre base/raiz de tronco vivo, 29/X/2004, L.D.P. Alvarenga 357 (MG).

Mesmo com todos os extensivos levantamentos, a riqueza e diversidade da Amazônia têm se mostrado inesgotável. A exemplo, Lisboa \& Ilkiu-Borges (1997) referiram 11 novas espécies para os estado do Pará e duas novas para o Brasil, e Ilkiu-Borges \& Lisboa (2004), referiram três para o Pará e três para o Brasil. Este último trabalho é parte da dissertação de mestrado da primeira autora na qual, além das mencionadas, são referidas duas novas ocorrências para o estado do Pará e quatro para o Brasil, sendo uma nova para a América do Sul e uma nova para a ciência. É oportuno salientar que a autora analisou apenas uma família de briófitas, Lejeuneaceae, que é a mais importante e bem representada nos ecossistemas tropicais (Gradstein \& Pócs 1989; Gradstein et al. 2001). Naturalmente, estudos incluindo todos os grupos podem revelar ainda muitas novidades.

Tendo em mente que estas foram resultantes de um breve inventário, o assinalamento destas novas referências para o Estado do Pará, portanto, ratifica a necessidade de estudos contínuos no bioma Floresta Amazônica. Conforme salientou Lisboa (1991), o estudo das briófitas na Amazônia é relativamente recente e necessita ser mais bem aprofundado.

\section{Agradecimentos}

As autoras agradecem a Maria Aparecida de Freitas do projeto TEAM (Tropical Ecology Assessement and Monitoring); à coordenação da Estação Científica Ferreira Penna e do Programa de Residência em Estudos Amazônicos nas pessoas de Alcir Favacho e Luiza Magali Henrique, respectivamente.

\section{Referências bibliográficas}

Bastos, C.J.P. 2004. Lejeuneaceae (Marchantiophyta) no estado da Bahia, Brasil. Tese de Doutorado, Instituto de Biociências, Universidade de São Paulo, São Paulo.

Bastos, C.J.P. \& Yano, O. 2003. New Records of the Genus Rectolejeunea (Lejeuneaceae) for the State of The Bahia, Brazil. Nova Hedwigia 76: 477-485.

Buck, W.R. \& Goffinet, B. 2000. Morphology and Classification of Mosses. Pp. 71-123. In: J. Shaw \& B. Goffinet (eds.). Bryophyte Biology. Cambridge: Cambridge University Press.
Crandall-Stotler, B. \& Stotler, R.E. 2000. Morphology and classification of Marchantiophyta. Pp. 21-70. In: J. Shaw \& B. Goffinet (eds.). Bryophyte Biology. Cambridge University Press, Cambridge.

Dauphin, G. 2003. Ceratolejeunea. Flora Neotropica Monograph 90: 1-86.

Evans, A.W. 1906. Hepaticae of Puerto Rico. IV. Cheilolejeunea, Rectolejeunea, Cystolejeunea and Pycnolejeunea. Bulletin of the Torrey Botanical Club 31: 183-226.

Fullford, M.H. 1968. Manual of leafy hepaticae of Latin America - Part III. Memoirs of The New York Botanical Garden 11: 277-392.

Germano, S.R. \& Pôrto, K.C. 2004. Novos Registros de Briófitas para Pernambuco, Brasil. Acta Botanica Brasilica 18: 343-350.

Gradstein, S.R. 1994. Lejeuneaceae; Ptychantheae, Brachiolejeuneae. Flora Neotropica, Monograph 62: 1-225.

Gradstein, S.R. \& Costa, D.P. 2003. Liverworts and Hornworts of Brazil. Memoirs of The New York Botanical Garden 88: $1-673$.

Gradstein, S.R. \& Pócs, T. 1989. Bryophytes. In: H. Lieth \& M.J.A. Werger (eds.). Tropical Rain Forest Ecosystems 16: 311-325, Amsterdam.

Gradstein, S.R.; Churchill, S.P. \& Salazar-Allen., N. 2001. Guide to the Bryophytes of Tropical America. Memoirs of The New York Botanical Garden 86: 1-577.

Grolle, R. \& Heinrichs, J. 1999. Redescription and synonymy of Plagiochila aerea Taylor 1846 (Hepaticae), first described as Lycopodium pinnatum by Lamarck 1792 . Nova Hedwigia 68: 511-525.

Heinrichs, J.; Gradstein, S.R. \& Grolle, R. 1998. A revision of the neotropical species of Plagiochila (Dumort.) Dumort. (Hepaticae) described by Olof Swartz. Journal of the Hattori Botanical Laboratory 85: 1-32.

Ilkiu-Borges, A.L. \& Lisboa, R.C.L. 2002a. Os Gêneros Cyrtolejeunea A. Evans e Drepanolejeunea Steph. (Lejeuneaceae) na Estação Científica Ferreira Penna (PA) e Novas Ocorrências. Boletim do Museu Paraense Emílio Goeldi, Série Botânica 18: 231-245.

Ilkiu-Borges, A.L. \& Lisboa, R.C.L. 2002b. Os Gêneros Leptolejeunea e Raphidolejeunea (Lejeuneaceae) na Estação Científica Ferreira Penna, Pará, Brasil. Acta Amazonica 32: 205-215.

Ilkiu-Borges, A.L. \& Lisboa, R.C.L. 2002c. Os Gêneros Lejeunea e Microlejeunea (Lejeuneaceae) na Estação Científica Ferreira Penna, Estado do Pará, Brasil, e Novas Ocorrências. Acta Amazonica 32: 541-553.

Ilkiu-Borges, A.L. \& Lisboa, R.C.L. 2002d. Lejeuneaceae (Hepaticae). cap 5. Pp. 399-419. In: P.L.B. Lisboa (org.). Caxiuanã: Populações Tradicionais, Meio Físico e Diversidade Biológica. Belém, Museu Paraense Emílio Goeldi.

Ilkiu-Borges, A.L. \& Lisboa, R.C.L. 2004. Cololejeuneae (Lejeuneaceae, Hepaticae) na estação Científica Ferreira Pena, Melgaço, PA, Brasil. Acta Botanica Brasilica 18: 887-902. 
Jovet-Ast, S. 1953. Lê genre Colura. Hépatiques. Lejeuneaceae, Diplasiae. Revue Bryologique et Lichénologique 22: 206-312.

Lisboa, P.L.B. (org.) 2002. Caxiuanã: populações tradicionais, meio físico e diversidade biológica. Belém, Museu Paraense Emílio Goeldi.

Lisboa, R.C.L. 1991. Histórico da briologia na Amazônia brasileira. Boletim do Museu Paraense Emílio Goeldi, Série Botânica 7: 69-77.

Lisboa, R.C.L. 1993. Musgos Acrocárpicos do Estado de Rondônia. Belém, Museu Paraense Emílio Goeldi.

Lisboa, R.C.L. 1994. Adições à brioflora do Estado do Pará. Boletim do Museu Paraense Emílio Goeldi 10: 15-42.

Lisboa, R.C.L. \& Ilkiu-Borges, A.L. 1997a. Novas ocorrências de Bryophyta (musgos) para o Estado do Pará, Brasil. Acta Amazonica 27: 81-102.

Lisboa, R.C.L. \& Ilkiu-Borges, A.L. 1997b. A Família Splacnobryaceae (Bryophyta) no Estado do Pará. Boletim do Museu Paraense Emílio Goeldi, Série Botânica 13: 103-111.

Lisboa, R.C.L. \& Ilkiu-Borges, A.L. 2001.Briófitas de São Luís do Tapajós, município de Itaituba, com novas adições para o Estado do Pará. Boletim do Museu Paraense Emílio Goeldi, Série Botânica 17: 75-91.

Lisboa, R.C.L. \& Lima, M.J.L. 1997. Leucophanaceae, nova família de Bryophyta para o Pará, Brasil. Acta Botanica Brasilica 11: 79-85.

Lisboa, R.C.L. \& Santos, R.C.P.2005a. Helicophyllaceae (Bryophyta), nova ocorrência para o Estado do Pará, Brasil. Acta Amazonica 36: 343-346.

Lisboa, R.C.L. \& Santos, R.C.P. 2005b. Ocorrência do Gênero Papillaria (Müll. Hal.) Müll. Hal. (Meteoriaceae, Bryophyta) na Amazônia. Boletim do Museu Paraense Emílio Goeldi, Série Ciências Naturais 1: 61-63.
Oliveira-e-Silva, M.I.M.N. 1998. Briófitas da Reserva Ecológica de Rio das Pedras, Município de Mangaratiba, do Parque Estadual da Ilhas Grande e da Reserva Biológica Estadual da Praia do Sul, Município de Angra dos Reis, Estado do Rio de Janeiro. Tese de doutorado. Universidade de São Paulo, Instituto de Biociências. São Paulo.

Osakada, A. \& Lisboa, R.C.L. 2004. Novas ocorrências de hepáticas (Marchantiophyta) para o Estado do Pará Brasil. Acta Amazonica 34: 197-200.

Pôrto, K.C. 1996. Briófitas. Pp. 91-103. In: E.V.S.B. Sampaio; S.J. Mayo \& M.R.V. Barbosa (eds.). Pesquisa Botânica Nordestina: Progresso e Perspectivas. Sociedade Botânica do Brasil, Regional Pernambuco, Recife.

Reiner-Drehwald, M.E. \& Goda, A. 2000. Revision of the genus Crossotelejeunea (Lejeuneaceae, Hepaticae). The Journal of the Hattori Botanical Laboratory 89: 1-54.

Souza, A.P.S \& Lisboa, R.C.L. 2005. Musgos (Bryophyta) na Ilha Trambioca, Barcarena, PA, Brasil. Acta Botanica Brasilica 19: 487-492.

Yano, O. 1981. A checklist of Brazilian Mosses. The Journal of the Hattori Botanical Laboratory 50: 279-456.

Yano, O. 1984. Checklist of Brazilian liverworts and hornworts. The Journal of the Hattori Botanical Laboratory 56: 481-548.

Yano, O. 1989. An additional checklist of Brazilian bryophytes. The Journal of the Hattori Botanical Laboratory 66: 371-434

Yano, O. 1995. A new additional annotated checklist of Brazilian bryophytes. The Journal of the Hattori Botanical Laboratory 78: 7-182. 